\title{
Editorial
}

\section{THE STIGMA OF LEPROSY}

It is trite to remark that the sufferer from leprosy has to bear a double burden-the disease itself, and the stigma that goes with it. In this issue of Leprosy Review contributors with different backgrounds examine the concept of stigma in leprosy, share their personal experiences of its reality, make suggestions for modifying or removing the disfiguring disabilities that characterize the disease, and, finally, enter an impassioned plea for the abolition of the term "leprosy" (and its cognates) in the hope that this will reduce its stigma.

Stigma is a complex thing. Originally, the word denoted marks made by a pointed instrument or a heated iron. It thus took on the meaning of branding of the skin of man or beast to indicate ownership or subjection. It could be a sign of guilt or disgrace, of infamy or shame. More recently, the root etymological meaning has been extended to embrace any departure from a physical norm, or any obvious defect suggestive of a certain condition or disease. A stigma is undesirable, reprehensible or objectionable. As used in regard to leprosy, stigma refers not only to the characteristic visible physical signs of paralysis, ulceration, and deformity associated with advanced peripheral neuropathy, but also by extension to the whole gamut of irrational fears and prejudices under which the leprosy victim suffers. Some folk even claim that leprosy has its own special "smell"-nauseating and repelling. The invisible can be more dreadful and more dreaded than the palpable-and equally stigmatizing.

The historical association of visible blemishes with ceremonial defilement also plays a part in the development or persistence of stigma in many cultures, and not only in the Judaeo-Christian. It is noteworthy that in the course of centuries an enhanced dread of "leprosy" has emerged pari passu with a diminution of the ritualistic "uncleanness" of, say, mildewed cloth or fungal plaques on damp walls. Yet for most people in the Western world, the stigma of leprosy depends less on personal encounters with the condition than on hearsay and folklore. Amongst the educated strata of society, not excepting medical men, a curious dichotomy of thought is frequently to be observed: there may be a conscious and intellectual acceptance of the scientific facts about leprosy, and at the same time a subconscious rejection of these facts in favour of traditional beliefs.

There are, of course, other conditions besides leprosy associated with stigma; for example, epilepsy, facial disfigurement from whatever cause, mental defect, and congenital deformities. Other groups of people suffer social or psychological disabilities to a degree that depends on subjective factors; blindness, for instance, enlists more sympathy than deafness, and the victim of poliomyelitis than someone suffering from a venereally-acquired disease. The degree of stigma may be partly correlated with nomenclature and semantics: the "lunatics" and "consumptives" of a former era certainly had their cross to bear. Some skin abnormalities may be stigmatizing by reason of diagnostic confusion or 
uncertainty: the "white leprosy" of India and countries of the Near East makes of vitiligo an abhorrent disease, and infuses psoriasis with an unwarranted morbidity and contagiousness.

Stigma in leprosy is determined by a wide range of changing variables. It may be completely absent in some communities where the prevalence is so high that "everybody gets these light patches on the skin sooner or later, but most of them go away on their own". Although no stigma may attach to the hypopigmented cutaneous lesion, the fear of the discharge from ulcerating extremities may be such that when that stage is reached the victim is abandoned in the bush to fend for himself, or to die. Similarly, with madarosis: to some, absence of eyebrows is a sign of active leprosy, and is feared; to others, accustomed or not to the cosmetic plucking of the eyebrow hairs, it carries no stigma. In countries where saddle-back nose is more frequently due to tertiary yaws than to leprosy, this deformity has no stigmatizing character. In some situations, other skin diseasesespecially if ulcerating, obvious, chronic, malodorous, or occurring on the face-may be more potentially stigmatizing than leprosy.

In some localities, other conditions which occur in people suffering from leprosy are part and parcel of the stigma of the disease, for example, enlarged cervical nerves, wasting of the first interosseous space, hanging ear-lobes, gynaecomastia, etc. They are recognized by the laity as due to leprosy, and frequently regarded moreover as evidence of active disease. Protective footwear and such appliances as foot-drop springs may stigmatize the wearer as suffering from leprosy.

If the stigma of leprosy varies with the population concerned, it is generally true that leprosy is characterized by a fear unparalleled in any other condition, and associated in the lay mind with the deformities of advanced nerve damage. Folklore lends widespread support to this view. The possibility of deformity, even the inevitability of deformity, looms large in the individual and corporate subconscious. The prospect that the disease will progress and lead sooner or later to social rejection, unemployability, starvation and physical suffering, is reinforced by observation and by communal lore. Even when treatment becomes available, these fears of recognizable stigmata of ten prove impossible to eradicate. Patients themselves may imagine slight departures from the normal in their physical appearance, and between them build up a corpus of beliefs about the stigma of their common disease.

Again, stigma in leprosy may be created de novo where it did not before exist, by the well-intentioned efforts of those concerned to do something for a neglected human problem. By concentrating on the relief of patients in one category-to the disregard of those suffering from other widespread endemic diseases or from malnutrition-such activities may inculcate the concept that leprosy is after all somehow different, and requires its own medical and organizational control procedures. Over-emphasis by visual misrepresentation or emotive phraseology may actually confirm prejudices, and so render the educative task of diminishing stigma the more difficult and protracted.

Voluntary agencies thus have a difficult rôle to play in their continuing and just concern that the victim of leprosy shall be regarded like any other hum an being, able to get treatment and employment. Their propaganda and fund-raising activities must eschew the easy way that may, as an undesirable side-effect, tend to increase the stigma (and hence the suffering and resentment) of those they purport to be helping. It is not easy, in focusing attention on a single problem, to 
avoid perpetuating the stigma of leprosy, just as the maintenance of specialized and rehabilitation services may have the same unwanted result. They should view their particular interest in the context of related medical and social needs. With equal cogency, it may be added that governments that subscribe to the Bill of Human Rights and supported the Human Rights Year sponsored by the United Nations might enquire if in law and practice there is in their countries any discrimination against those who suffer from leprosy, whether or not they bear any disfiguring marks attributable to the disease.

Personal observation reinforces information from reliable sources that to this day in some countries the branding of any citizen as a "leper" in practice automatically means that he is deprived of all human rights-to hold property, to marry or remain married, to work, to live where he wishes, to obtain medical treatment. He is deprived of liberty and livelihood. Stigma means cruelty and suffering.

For some, stigma resides rather in words than in the disease itself, more in the accumulated pejorative connotations of "leprosy" than in the actual physical manifestations of the disease or its sequelae. The weight of this argument is felt particularly by our South American colleagues. Perhaps the word "leprosy" needs rehabilitating, deprived of all stigmatizing content and given international respectability. If we could by education remove the accidentia of the word "leprosy", perhaps the stigma would go and the social part of our task would lessen.

\section{... "That which we call a rose}

By any other name would smell as sweet."

As Dean Swift so rightly enquired, "If the physicians would forbid us to pronounce the words [ of certain diseases], would that expedient serve like so many talismen to destroy the diseases themselves?" The epithet gets attached to a person of normal appearance, who at once becomes invested with the imagined signs of advanced disease. The label sticks. The dog is given a bad name, whether it deserves it or not. The word "leper" is officially banned; should not "leprosy" go the same way?

Another area of conflicting assumptions related to the stigma of leprosy is concerned with the increasing secularization of the disease. As in many countries and in many cultures the traditional association of leprosy and Divine displeasure becomes more tenuous and less tenable, so the aura of mystique will diminish and the linking of stigmatizing deformity with morality and punishment will tend to disappear. Voluntary agencies, which still account for a much-appreciated proportion of funds and service devoted to leprosy and leprosy sufferers, should be relating their publicity and fund-raising activities to the changing situation if their appeal is to retain its credibility and impact. Similarly, segregation of patients in leprosaria for long periods may perpetuate the stigma as well as constitute a permanent charge on government or donor agency.

Sir Winston Churchill's fine dictum about crime and criminals might with some justice be adapted to stigma in leprosy: "The mood and temper of the public in regard to" its attitude to leprosy and those who suffer from leprosy "is one of the most unfailing tests of the civilization of any country".

This, then, is the situation as depicted in the articles that follow, and this is the task to which all who work in leprosy must not fail to address themselves. To diminish fear, to remove prejudice, to educate the public (not forgetting the 
doctors, the politicians, and the rising generation), to mitigate surgically the physical bases of stigma, and to prevent their occurrence by proper medical treatment-these together should reduce the burden of stigma which far too many leprosy sufferers are still compelled by society to carry. 